J. Piprek (ed.): "Optoelectronic Devices:

Advanced Simulation and Analysis" Springer, 2005

\title{
14 Monolithic Wavelength Converters: Many-Body Effects and Saturation Analysis
}

\author{
J. Piprek ${ }^{1}$, S. Li ${ }^{2}$, P. Mensz ${ }^{2}$, and J. Hader ${ }^{3}$ \\ 1 University of California, Santa Barbara, CA 93106-9560, U.S.A., \\ piprek@ieee.org \\ 2 Crosslight Software, 202-3855 Henning Dr., Burnaby, BC V5C 6N3 Canada, \\ simon@crosslight.com \\ 3 Nonlinear Control Strategies, 1001 East Rudasill Rd., Tucson, AZ 85718, \\ U.S.A., jhader@acms.arizona.edu
}

\subsection{Introduction}

Wavelength converters are a novel class of photonic integrated circuits that is crucial for multiwavelength fiber-optic communication networks [1]. Such converters switch the flow of information from one wavelength to another. We present here the simulation and analysis of an optoelectronic InP-based tunable wavelength converter (Fig. 14.1) that monolithically combines a preamplified receiver with a postamplified sampled-grating distributed Bragg reflector (SG-DBR) laser diode [2]. We employ the commercial software PICS3D [3], which was modified for the purpose of this investigation. Our self-consistent physical model takes into account many-body gain and absorption in the quantum wells, carrier drift and diffusion, and optical waveguiding. The time-consuming calculation of many-body spectra is performed externally [4], based on the theory outlined in Chap. 1 and in [5]. Tabulated spectra of gain, spontaneous emission, and index change are then imported into PICS3D. Performance limitations by saturation effects are the main target of this investigation.

The next section outlines the device structure. Section 14.3 describes physical device models and material parameters, including their experimental calibration. The following Sect. 14.4 investigates each component of the wavelength converter by three-dimensional (3D) steady-state simulation. Timedomain simulations of a similar SG-DBR laser can be found in Chap. 6 .

\subsection{Device Structure}

Figure 14.1 gives the schematic design of the wavelength converter [6]. It electrically couples an optical receiver for any input wavelength of the $C$-band, e.g., $\lambda_{\text {in }}=1525 \mathrm{~nm}$, with an optical transmitter for any output wavelength of the $C$-band, e.g., $\lambda_{\text {out }}=1565 \mathrm{~nm}$. The receiver integrates signal preamplification by a semiconductor optical amplifier (SOA) and signal detection by a waveguide photodiode (WPD). The optical signal is converted into an 


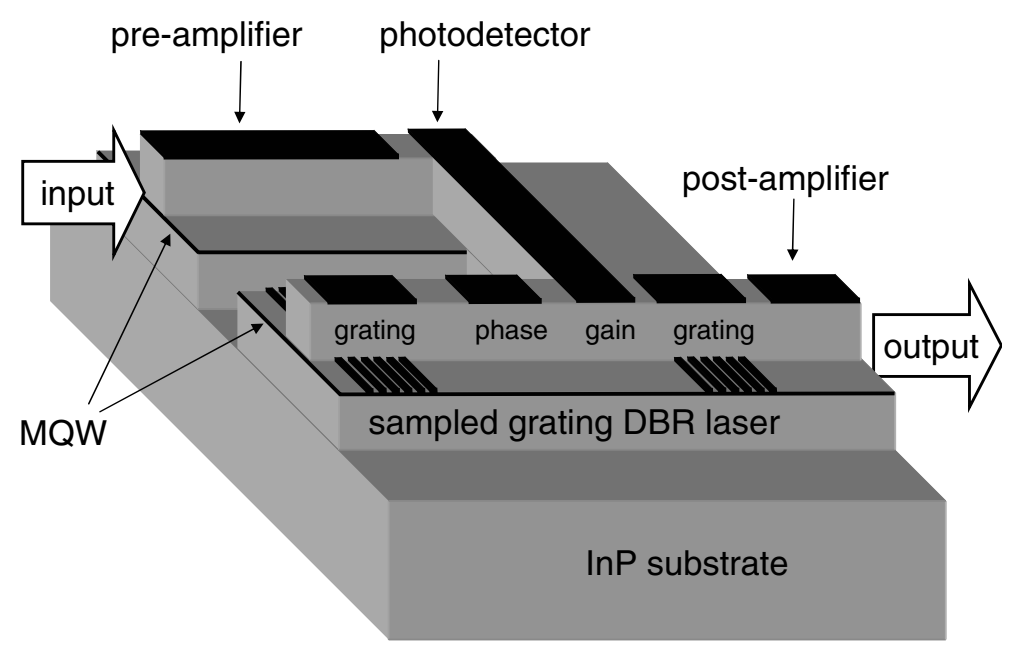

Fig. 14.1. Schematic view of the monolithic wavelength converter.

electrical signal that directly modulates a SG-DBR laser diode that is integrated with a semiconductor optical amplifier for signal enhancement [7]. The SG-DBR laser can be widely tuned to emit at any wavelength of the $C$-band [8].

The device is grown on $\mathrm{InP}$ by metal organic chemical vapor deposition; details of the fabrication process are given in [2]. The layer structure of the different components is very similar as all are based on the same epitaxial growth (Table 14.1). An offset-multi-quantum-well (MQW) active region is grown on top of the waveguide layer. For lateral confinement of optical field and current flow, a narrow ridge-waveguide structure is etched down to the MQW region. Passive device sections are formed by etching off the MQW.

\subsection{General Device Physics}

The different device types integrated in the wavelength converter (laser, amplifier, detector) exhibit the same epitaxial layer structure as well as some common device physics, which is discussed in this section. Material parameters are listed in Table 14.2 for binary compounds. They are linearly interpolated to obtain InGaAsP parameters, unless noted otherwise in the following.

\subsubsection{Optical Waveguiding}

The software solves the scalar Helmholtz equation:

$$
\frac{\partial^{2} \Phi}{\partial x^{2}}+\frac{\partial^{2} \Phi}{\partial y^{2}}+\left(k^{2}-\beta^{2}\right) \Phi=0,
$$


Table 14.1. Epitaxial Layer Structure [9] (intrinsic $i$-regions exhibit about $10^{16} \mathrm{~cm}^{-3} n$-type background doping).

\begin{tabular}{|c|c|c|c|}
\hline & $\begin{array}{l}\text { Thickness } \\
(\mathrm{nm})\end{array}$ & $\begin{array}{l}\text { Doping } \\
\left(1 / \mathrm{cm}^{3}\right)\end{array}$ & $\begin{array}{l}\text { Mobility }(n / p) \\
\left(\mathrm{cm}^{2} / \mathrm{Vs}\right)\end{array}$ \\
\hline$p$-InGaAs contact & 100 & $3 \times 10^{19}$ & $100 / 20$ \\
\hline$p$-InP cladding & 1600 & $1 \times 10^{18}$ & $2200 / 70$ \\
\hline$p$-InP cladding & 200 & $3 \times 10^{17}$ & $2800 / 100$ \\
\hline$i$-InP doping setback & 50 & - & $4300 / 160$ \\
\hline$i-\operatorname{In}_{0.735} \mathrm{Ga}_{0.265} \mathrm{As}_{0.513} \mathrm{P}_{0.487}$ barrier $(7)$ & 8 & - & $4300 / 160$ \\
\hline$i-\operatorname{In}_{0.735} \mathrm{Ga}_{0.265} \mathrm{As}_{0.845} \mathrm{P}_{0.155}$ well $(7)$ & 6.5 & - & $4300 / 160$ \\
\hline$i-\operatorname{In}_{0.735} \mathrm{Ga}_{0.265} \mathrm{As}_{0.513} \mathrm{P}_{0.487}$ barrier & 8 & - & $4300 / 160$ \\
\hline$i$-InP etch stop & 10 & - & $4300 / 160$ \\
\hline$n$ - $\mathrm{In}_{0.612} \mathrm{Ga}_{0.338} \mathrm{As}_{0.728} \mathrm{P}_{0.272}$ waveguide & 350 & $1 \times 10^{17}$ & $3300 / 130$ \\
\hline$n$-InP cladding & 1400 & $1 \times 10^{18}$ & $2200 / 70$ \\
\hline$n$-InGaAs contact & 100 & $1 \times 10^{18}$ & $2200 / 70$ \\
\hline$i$-InP buffer & 1000 & - & $4300 / 160$ \\
\hline
\end{tabular}

Table 14.2. Binary Material Parameters.

\begin{tabular}{lllrrrr}
\hline Parameter & \multicolumn{2}{l}{ Symbol Unit } & GaAs & GaP & InAs & InP \\
\hline Direct band gap (unstrained) & $E_{\mathrm{g}}$ & $\mathrm{eV}$ & 1.423 & 2.773 & 0.356 & 1.35 \\
Spin-orbit split energy & $\Delta_{0}$ & $\mathrm{eV}$ & 0.341 & 0.080 & 0.410 & 0.11 \\
Electron eff. mass & $m_{\mathrm{c}}$ & $m_{0}$ & 0.0665 & 0.131 & 0.027 & 0.064 \\
Luttinger parameter & $\gamma_{1}$ & - & 6.85 & 4.20 & 19.67 & 6.35 \\
Luttinger parameter & $\gamma_{2}$ & - & 2.10 & 0.98 & 8.37 & 2.08 \\
Luttinger parameter & $\gamma_{3}$ & - & 2.90 & 1.66 & 9.29 & 2.76 \\
Lattice constant & $a_{0}$ & $\AA$ & 5.65325 & 5.451 & 6.0583 & 5.869 \\
Elastic constant & $C_{11}$ & $10^{11} \mathrm{dyn} / \mathrm{cm}^{2}$ & 11.81 & 14.12 & 8.329 & 10.22 \\
Elastic constant & $C_{12}$ & $10^{11} \mathrm{dyn} / \mathrm{cm}^{2}$ & 5.32 & 6.253 & 4.526 & 5.76 \\
Elastic constant & $C_{44}$ & $10^{11} \mathrm{dyn} / \mathrm{cm}^{2}$ & 5.94 & 7.047 & 3.959 & 4.60 \\
Hydrost. deformation potential & $a_{1}$ & $\mathrm{eV}$ & -7.1 & -5.54 & -5.9 & -6.35 \\
Shear deformation potential & $a_{2}$ & $\mathrm{eV}$ & -1.7 & -1.6 & -1.8 & -2.0 \\
Dipole matrix energy & $E_{p}$ & $\mathrm{eV}$ & 22.80 & 21.51 & 14.72 & 15.22 \\
LO phonon energy & $\hbar \omega$ & $\mathrm{eV}$ & 0.0354 & 0.046 & 0.0296 & 0.0426 \\
opt. dielectric constant & $\varepsilon_{\infty}$ & - & 10.9 & 9.075 & 12.25 & 9.61 \\
stat. dielectric constant & $\varepsilon_{\text {st }}$ & - & 12.91 & 11.1 & 15.15 & 12.61 \\
refractive index parameter & $A_{\mathrm{r}}$ & - & 6.30 & 22.25 & 5.14 & 8.40 \\
refractive index parameter & $B_{\mathrm{r}}$ & - & 9.40 & 0.90 & 10.15 & 6.60 \\
\hline
\end{tabular}


where $\Phi(x, y)$ represents any transverse component of the optical field, $k$ is the absolute value of the wavevector, and $\beta$ is the longitudinal propagation constant. The calculated vertical intensity profile is plotted in Fig. 14.2 together with the refractive index profile. The nonsymmetric index profile results in a reduced optical confinement factor for the quantum wells of $\Gamma=0.06$. The 2D profile of the fundamental optical mode is given in Fig. 14.3 for half the device cross section. It is well confined by the $p$-InP ridge. The narrow ridge width $W=3 \mu \mathrm{m}$ prevents the appearance of higher-order lateral modes, as confirmed by measurements.

In propagation direction of the optical wave ( $z$ axis), the modal optical intensity $P_{\mathrm{m}}(z)$ changes according to:

$$
\frac{d P_{\mathrm{m}}}{d z}=\left(g_{\mathrm{m}}-\alpha_{m}\right) P_{\mathrm{m}},
$$

with $g_{\mathrm{m}}(z)$ giving the modal optical gain or absorption due to band-to-band transitions within the quantum well (see next section) and $\alpha_{\mathrm{m}}(z)$ giving the modal optical loss caused by other processes.

Near $1.55-\mu \mathrm{m}$ wavelength, optical losses are mainly attributed to intervalence band absorption (IVBA). The IVBA coefficient is considered proportional to the local hole density, i.e., it is only relevant within the quantum wells and within p-doped regions. The local loss coefficient is calculated as:

$$
\alpha_{\mathrm{opt}}=\alpha_{\mathrm{b}}+k_{n} n+k_{p} p,
$$

with the background absorption $\alpha_{\mathrm{b}}$, the electron density $n$, and the hole density $p$. The hole coefficient $k_{p}=25 \times 10^{-18} \mathrm{~cm}^{2}$ [10], the electron coefficient $k_{n}=1 \times 10^{-18} \mathrm{~cm}^{2}$, and $\alpha_{\mathrm{b}}=9 \mathrm{~cm}^{-1}$ are employed in our simulations. The background loss is mainly related to photon scattering. The modal loss coefficient $\alpha_{\mathrm{m}}(z)$ is obtained by $2 \mathrm{D}$ integration over $\alpha_{\mathrm{opt}}(x, y, z)$ within each $x y$ cross section, weighted by the optical intensity $P_{\text {opt }}(x, y, z)$.

The InGaAsP refractive index $n_{\mathrm{r}}$ is calculated as function of the photon energy $h \nu$ using:

$$
n_{\mathrm{r}}^{2}(h \nu)=A_{\mathrm{r}}\left[f\left(x_{1}\right)+0.5\left(\frac{E_{\mathrm{g}}}{E_{\mathrm{g}}+\Delta_{0}}\right)^{1.5} f\left(x_{2}\right)\right]+B_{\mathrm{r}}
$$

with:

$$
\begin{array}{ll}
f\left(x_{1}\right)=\frac{1}{x_{1}^{2}}\left(2-\sqrt{1+x_{1}}-\sqrt{1-x_{1}}\right), \quad x_{1}=\frac{h \nu}{E_{\mathrm{g}}} \\
f\left(x_{2}\right)=\frac{1}{x_{2}^{2}}\left(2-\sqrt{1+x_{2}}-\sqrt{1-x_{2}}\right), \quad x_{2}=\frac{h \nu}{E_{\mathrm{g}}+\Delta_{0}},
\end{array}
$$

which was shown to give good agreement with measurements on InGaAsP by linear interpolation of the binary material parameters $A_{\mathrm{r}}$ and $B_{\mathrm{r}}$ (Table 14.2) [11]. 


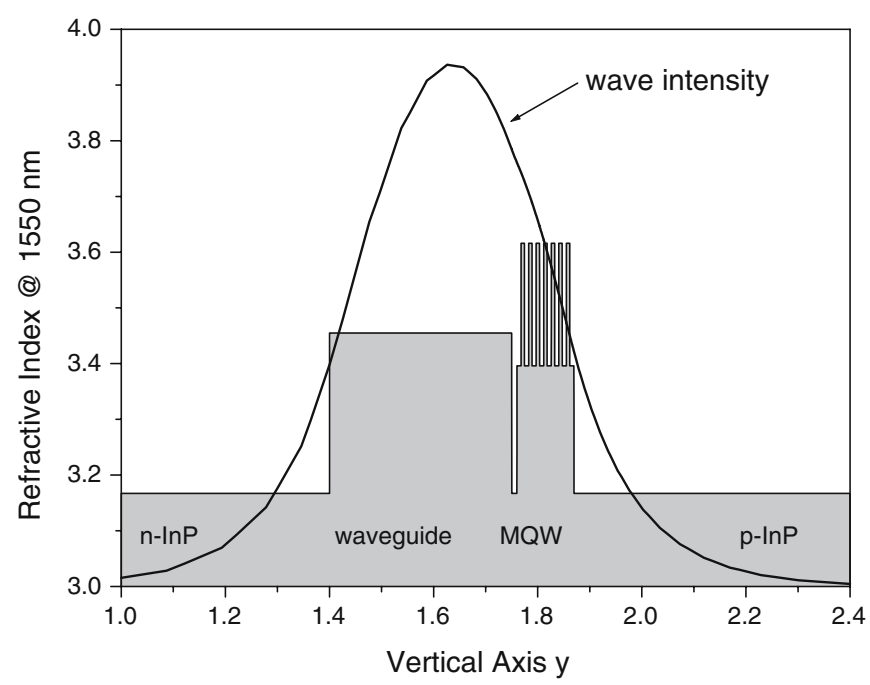

Fig. 14.2. Vertical profile of refractive index and wave intensity in the center of the device ( $\mathrm{y}$ in microns).

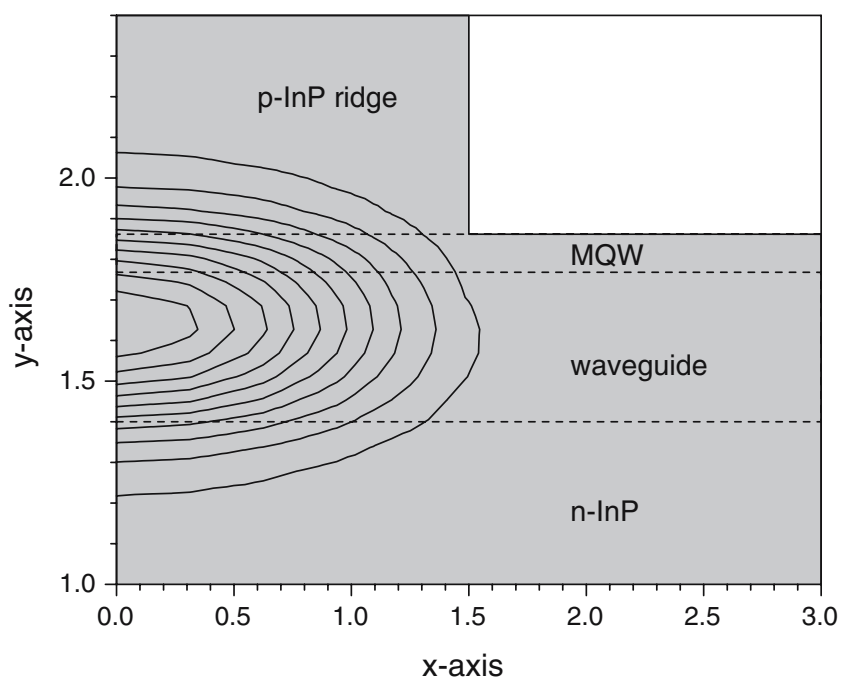

Fig. 14.3. 2D profile of the optical mode for half the device (x, y in microns). 


\subsubsection{Quantum Well Active Region}

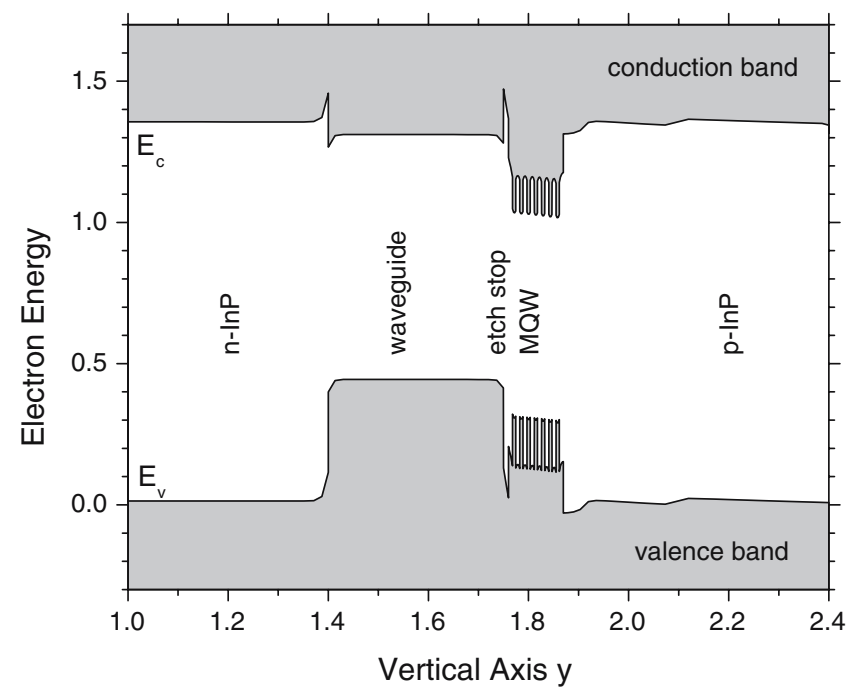

Fig. 14.4. Energy band diagram (electronvolts) for the active region at forward bias $\left(E_{\mathrm{c}}\right.$ - conduction band edge, $E_{\mathrm{v}}$ - valence band edge, y in microns).

Figure 14.4 shows the energy band diagram of the MQW active region. The unstrained $\mathrm{In}_{1-x} \mathrm{Ga}_{x} \mathrm{As}_{y} \mathrm{P}_{1-y}$ band gap is given in electronvolts by [12]:

$$
E_{\mathrm{g}}=1.35-0.775 y+0.149 y^{2} .
$$

The conduction band edge offset ratio $\Delta E_{\mathrm{c}} / \Delta E_{\mathrm{g}}=0.4$ is employed at all interfaces, which was demonstrated to give good agreement with measurements on similar devices [13].

PICS3D calculates the energy band structure of quantum wells, including strain effects using the $4 \times 4 \boldsymbol{k} \cdot \boldsymbol{p}$ model as published by Chuang [12]. By default, it then uses a free-carrier model to account for radiative carrier recombination within the quantum wells. However, previous investigations have shown that such free-carrier model results in poor agreement with measurements [9]. The many-body model outlined in the first chapter of this book is expected to more accurately describe optical spectra of quantum wells. The correct representation of the entire gain spectrum is of particular importance in our multiwavelength device. Therefore, we here use many-body spectra calculated for our quantum wells and imported into PICS3D. These 
computations are based on $8 \times 8 \boldsymbol{k} \cdot \boldsymbol{p}$ bandstructure calculations using the parameters listed in Table 14.2.

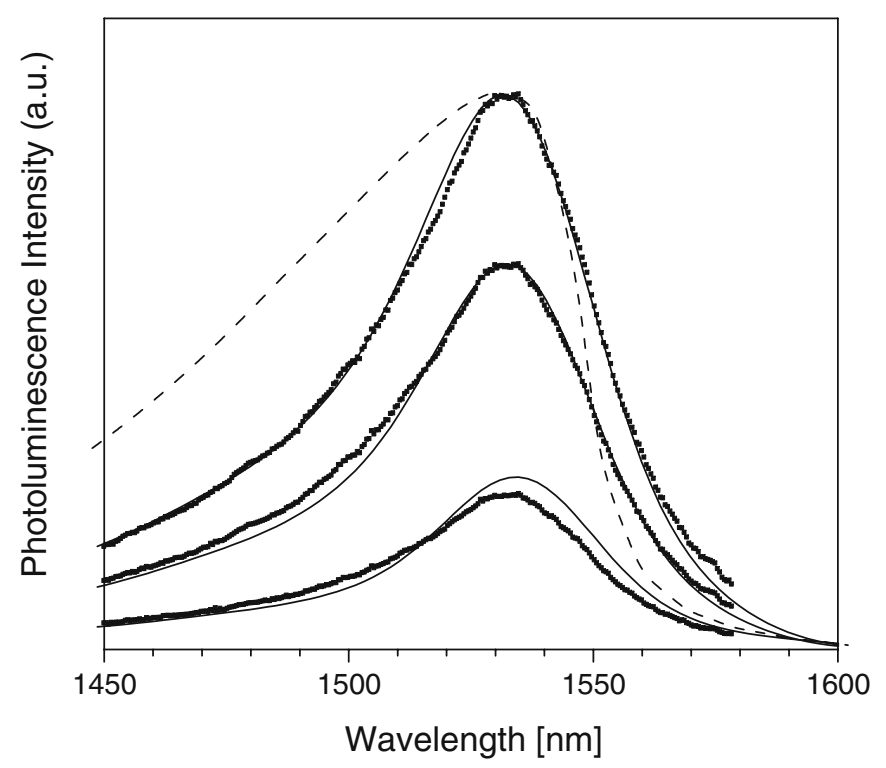

Fig. 14.5. Photoluminescence spectra of our MQW active region (dots - measured at $1 \times, 2 \times$, and $3 \times$ pump intensity [14], solid lines - many-body calculations at carrier densities of $3.8,6.2$, and $7.7 \times 10^{17} \mathrm{~cm}^{-3}$, dashed line - normalized freecarrier spectrum).

The dots in Fig. 14.5 show measured photoluminescence (PL) spectra. The emission peak at $1532 \mathrm{~nm}$ indicates a slight growth deviation from the intended MQW composition (cf. Table 14.1). The PL peak wavelength can be matched in the simulation by adjustment of the MQW composition, maintaining the measured biaxial MQW strain: $0.6 \%$ compressive strain in the quantum wells and $0.3 \%$ tensile strain in the barriers. This way, we obtain $\mathrm{In}_{0.685} \mathrm{Ga}_{0.315} \mathrm{As}_{0.864} \mathrm{P}_{0.136}$ for the quantum well composition and $\mathrm{In}_{0.685} \mathrm{Ga}_{0.315} \mathrm{As}_{0.595} \mathrm{P}_{0.405}$ for the barrier composition. The adjustment may also reflect inaccuracies in the calculation of the strained quaternary energy band gap. The only unknown parameter of the many-body calculation is the inhomogeneous energy broadening due to structural imperfections of the quantum well. A full-width half-maximum (FWHM) value of $14 \mathrm{meV}$ gives good agreement with the PL measurement (solid lines in Fig. 14.5). The quan- 
tum well carrier density is varied in the simulation to fit the measurement. For comparison, the dashed line shows the normalized free-carrier spectrum with homogeneous broadening of $13.6 \mathrm{meV}$ (scattering time $=0.1 \mathrm{ps}$ ). This parameter can be adjusted to find a better fit on the long-wavelength side, however, the PL intensity remains overestimated on the short wavelength side [9].

The corresponding many-body gain spectra are plotted in Fig. 14.6, and the gain at fixed wavelengths is given in Fig. 14.7 as a function of carrier density. The wavelength range of interest $(1525-1565 \mathrm{~nm})$ is completely covered by positive gain for carrier densities above $3 \times 10^{18} \mathrm{~cm}^{-3}$. The gain spectrum flattens at high densities. At very low carrier density, the manybody absorption spectrum shows a characteristic exciton peak. For comparison, free-carrier gain and absorption spectra are given by dashed lines as calculated with the $4 \times 4 \boldsymbol{k} \cdot \boldsymbol{p}$ band structure model, including valence band mixing. There are significant differences between many-body and free-carrier spectra, which are discussed in the following. At low carrier density, the exciton absorption peak is missing in the free-carrier spectrum because Coulomb attraction between electrons and holes is not considered. At high densities, the free-carrier gain peaks at shorter wavelength due to the missing band gap renormalization caused by many-body interaction. The larger width of the free-carrier gain spectrum is attributed to the smaller density of states resulting from the $4 \times 4 \boldsymbol{k} \cdot \boldsymbol{p}$ model as compared with the $8 \times 8 \boldsymbol{k} \cdot \boldsymbol{p}$ model used in the many-body calculation. Thus, the separation $\Delta E_{\mathrm{F}}$ of the quasiFermi levels needs to be wider in the free-carrier model to accommodate the same number of carriers in the quantum well. The larger magnitude of the free-carrier gain is unexpected because Coulomb interaction is known to cause gain enhancement [15]. The gain magnitude is proportional to the bulk matrix element:

$$
M_{\mathrm{b}}^{2}=\frac{m_{0}}{6} E_{p},
$$

with the electron rest mass $m_{0}$ and the material parameter $E_{p}$, which is interpolated between the binary data given in Table 14.2. Linear interpolation results in $E_{p}=17.3 \mathrm{eV}$ for our quantum well as used in both the freecarrier and the many-body model. The recent compilation of band structure parameters in [16] suggests a larger number of $E_{p}=24 \mathrm{eV}$ for our case; however, validation of either number requires absorption measurements that are not available for our quantum well material. Thus, some uncertainty remains with the magnitude of gain and absorption in our quantum wells.

The quantum well gain at given carrier density and wavelength tends to decrease at very high photon densities $S$. Such nonlinear behavior (gain compression) is attributed to the depletion of carriers at the transition energy level. This spectral hole burning is due to the finite time required for electrons and holes to fill up the energy levels emptied by stimulated recombination 


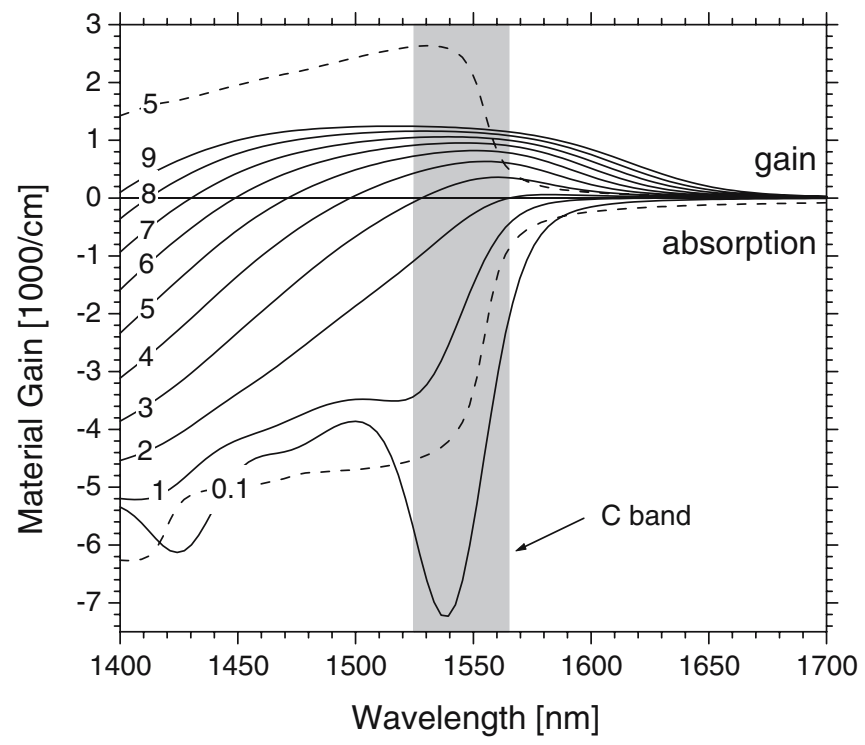

Fig. 14.6. Many-body gain spectra with the quantum well carrier density $n=p$ given as parameter $\left(\times 10^{18} \mathrm{~cm}^{-3}\right)$. The dashed lines show the free-carrier spectrum at two densities.

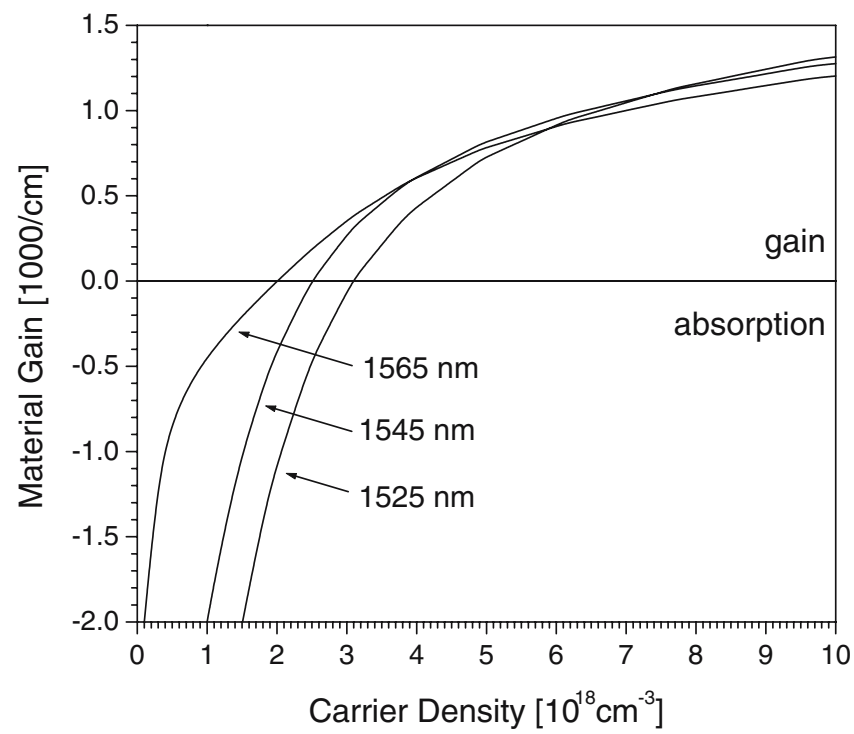

Fig. 14.7. Many-body gain vs. quantum well carrier density at three fixed wavelengths of the $C$-band. 
and to reestablish a Fermi distribution. The nonlinear gain is commonly approximated by the phenomenological equation:

$$
g_{\mathrm{opt}}=\frac{g_{0}}{1+\epsilon S},
$$

with the linear gain $g_{0}$ and the gain compression coefficient $\epsilon \approx 10^{-17} \mathrm{~cm}^{3}$. This nonlinear gain formula is included in our model; however, the effect on our steady-state results is negligibly small because the photon density remains below $10^{15} \mathrm{~cm}^{-3}$. Note that a different form of gain saturation is due to the reduction of the total carrier density by stimulated recombination, which is included self-consistently in our model and which shows strong effects on our results.

\subsubsection{Carrier Transport}

Drift and diffusion of electrons and holes is calculated by solving the semiconductor transport equations. The current densities are given by:

$$
\begin{aligned}
& \boldsymbol{j}_{n}=q \mu_{n} n \boldsymbol{F}+q D_{n} \nabla n \\
& \boldsymbol{j}_{p}=q \mu_{p} p \boldsymbol{F}-q D_{p} \nabla p,
\end{aligned}
$$

with the elementary charge $q$ and the mobilities $\mu_{n}$ and $\mu_{p}$. The dopingdependent low-field mobilities listed in Table 14.1 are obtained from [17] using InP values also for InGaAsP due to the lack of experimental data for our quaternary layers. The diffusion constants $D_{n}$ and $D_{p}$ are replaced by mobilities using the Einstein relation $D=\mu k_{\mathrm{B}} T / q$ with the Boltzmann constant $k_{\mathrm{B}}$ and the temperature $T$. The electrostatic field $\boldsymbol{F}$ is calculated from the charge distribution by the Poisson equation:

$$
\nabla \cdot\left(\varepsilon_{\mathrm{st}} \varepsilon_{0} \boldsymbol{F}\right)=q\left(p-n+p_{\mathrm{D}}-n_{\mathrm{A}}\right)
$$

( $\varepsilon_{\mathrm{st}} \varepsilon_{0}$ - electrical permittivity, $p_{\mathrm{D}}, n_{\mathrm{A}}$ - donor and acceptor concentration). In our steady-state simulation, the continuity equations:

$$
\begin{gathered}
\nabla \cdot \boldsymbol{j}_{n}-q R=0 \\
-\nabla \cdot \boldsymbol{j}_{p}-q R=0
\end{gathered}
$$

describe the influence of carrier generation $(R<0)$ and recombination $(R>$ $0)$. Emission and absorption of photons in the quantum wells was discussed in the previous section. The total local recombination rate is:

$$
R=R_{\text {stim }}+R_{\text {spon }}+R_{\text {Auger }}+R_{\mathrm{SRH}},
$$

with the stimulated recombination rate:

$$
R_{\mathrm{stim}}=g_{\mathrm{opt}} \frac{P_{\mathrm{opt}}}{h \nu},
$$


the spontaneous emission rate outside the quantum wells: ${ }^{4}$

$$
R_{\mathrm{spon}}=B\left(n p-n_{\mathrm{i}}^{2}\right),
$$

the Auger recombination rate:

$$
R_{\text {Auger }}=\left(C_{n} n+C_{p} p\right)\left(n p-n_{\mathrm{i}}^{2}\right),
$$

and the defect-related Shockley-Read-Hall (SRH) recombination rate:

$$
R_{\mathrm{SRH}}=\frac{n p-n_{\mathrm{i}}^{2}}{\tau_{p}^{\mathrm{SRH}}\left(n+N_{\mathrm{c}} \exp \left[\frac{E_{\mathrm{t}}-E_{\mathrm{c}}}{k_{\mathrm{B}} T}\right]\right)+\tau_{n}^{\mathrm{SRH}}\left(p+N_{\mathrm{v}} \exp \left[\frac{E_{\mathrm{v}}-E_{\mathrm{t}}}{k_{\mathrm{B}} T}\right]\right)}
$$

$\left(n_{\mathrm{i}}\right.$ - intrinsic carrier density, $N_{\mathrm{c}, \mathrm{v}}$ - density of states of conduction, valence band; $E_{\mathrm{t}}$ - mid-gap defect energy). For the radiative coefficient, we assume a typical value of $B=10^{-10} \mathrm{~cm}^{3} \mathrm{~s}^{-1}$ and for the SRH lifetimes, $\tau_{n}^{\mathrm{SRH}}=\tau_{p}^{\mathrm{SRH}}=20 \mathrm{~ns}$. There is some uncertainty with these recombination parameters; however, Auger recombination is known to have the strongest impact on InP-based devices and we use the Auger parameter $C_{n}$ as fit parameter to find agreement with the measured threshold current. Auger recombination within the valence band is believed to be negligible in our device $\left(C_{p}=0\right)$ [18]. Figure 14.8 compares simulated light-current characteristics with the measurement on broad-area Fabry-Perot lasers [9]. The fit results in $C_{n}=10^{-29} \mathrm{~cm}^{6} \mathrm{~s}^{-1}$, which is one order of magnitude smaller than the value obtained in [9] using a free-carrier gain model. This difference can be easily understood. The lower many-body gain requires a higher quantum well carrier density to reach lasing threshold. In order to obtain the same threshold current, the Auger recombination needs to be scaled down. However, our Auger coefficient is still within the rather wide range of values reported in the literature [19].

PICS3D obtains quasi-3D solutions to the transport equations by slicing the device into many transversal $x y$ sections. Within each $2 \mathrm{D}$ section, the equations are solved self-consistently and the solutions are iteratively adjusted to the longitudinal photon density $S(z)$. Longitudinal variations of, e.g., the quantum well carrier density are accounted for this way; however, carrier flow in $z$ direction is not considered.

\subsection{Simulation Results}

This section investigates device physics and performance of each converter component. We mainly focus on saturation effects in amplifier and photodetector, which limit the device performance. Experimental device characteristics from the wafer used for the PL calibration in Fig. 14.5 are not yet available for comparison.

\footnotetext{
${ }^{4}$ Inside the quantum wells, the many-body luminescence spectrum is integrated over all photon energies.
} 


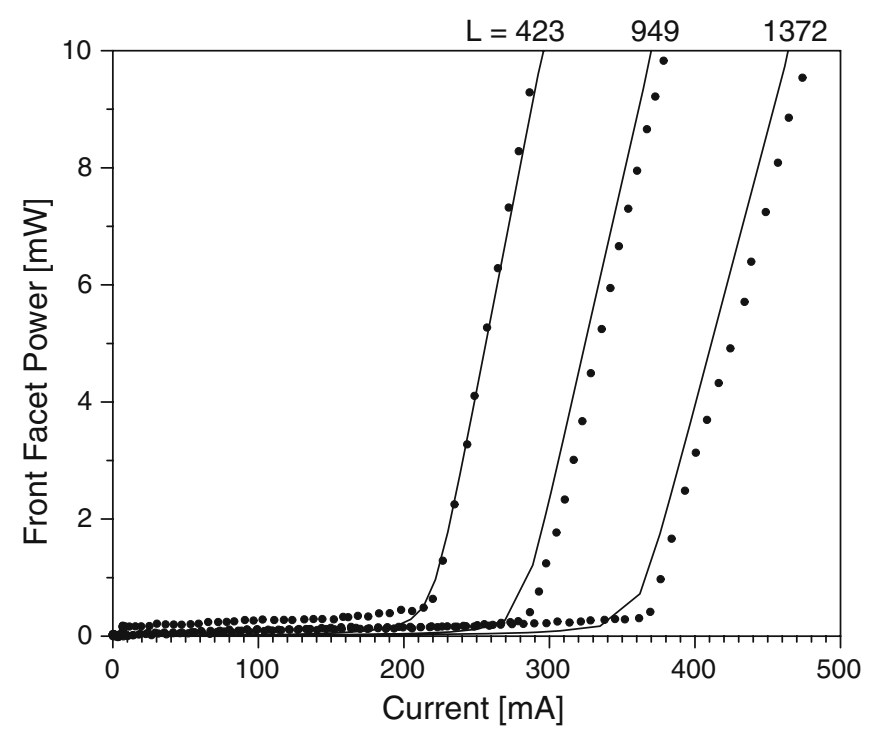

Fig. 14.8. Light-current (LI) curves for 50- $\mu$ m-wide Fabry-Perot lasers of different length $L / \mu \mathrm{m}$ (dots - measurement [9], lines - simulation with $C_{n}=$ $\left.10^{-29} \mathrm{~cm}^{6} \mathrm{~s}^{-1}\right)$.

\subsubsection{Amplifier}

Amplification enhances the optical signal, and it compensates for any losses during wavelength conversion. The SOA should provide maximum signal gain while maintaining linearity between input and output signal. Amplifier saturation results in a sublinear SOA response, and it restricts the maximum output signal.

We consider the ideal case of zero facet reflectance and maximum fiberto-waveguide power coupling efficiency of 0.25 , as estimated from the modal overlap between fiber and waveguide with perfect alignment. The coupling efficiency may be lower in the actual measurement [9]. The amplifier length is $600 \mu \mathrm{m}$ in our simulation.

At low input power, the material properties of the SOA are not affected by the amplified light. For such a case, Fig. 14.9 shows the SOA output power as a function of the injection current at different wavelengths. The wavelength dependence can be understood from the gain plots in Figs. 14.6 and 14.7. Power saturation at higher currents is caused by the sublinear dependence of the quantum well gain on current and carrier density. The red-shift of the gain spectrum with higher carrier density gives maximum gain at the longest wavelength for low currents and at the shortest wavelength for high 


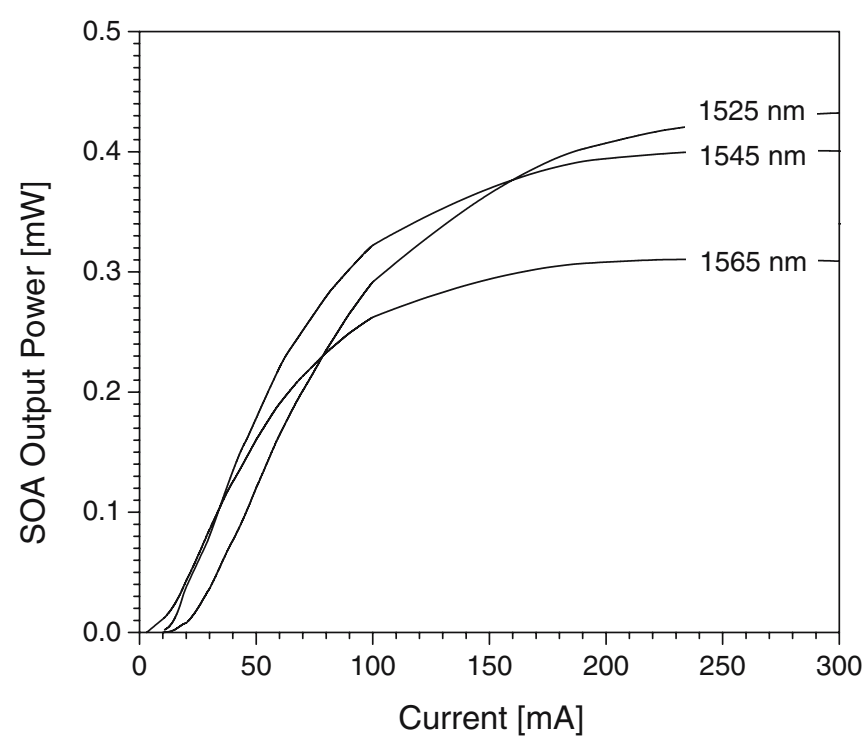

Fig. 14.9. SOA output power vs. injection current with the signal wavelength given as parameter (fiber input power $=0.1 \mathrm{~mW}$ ).

currents. Self-heating of the SOA would cause an additional red-shift of the gain spectrum, and it would lower the gain magnitude, thereby increasing gain saturation. However, the temperature of the wavelength converter is typically stabilized by a thermoelectric cooler [2]. The gain saturation with higher current restricts the maximum SOA gain, but it hardly affects the optical linearity of the SOA, which is investigated in the following.

With higher input power, the increasing modal intensity $P_{\mathrm{m}}(z)$ leads to increasing stimulated carrier recombination, which reduces the carrier density in the quantum wells as well as the modal gain toward the output facet. Figure 14.10 visualizes longitudinal variations of electron density, modal gain, and modal power for different injection currents. At low current $(15 \mathrm{~mA})$, the electron density is not high enough for amplification because the active region still exhibits net absorption (including optical losses), leading to a decay of the traveling optical wave. At higher current, positive net modal gain causes the light power to increase with travel distance. If the input power is high enough, the photon-triggered stimulated recombination reduces the carrier density with travel distance, thereby lowering the local gain. High modal intensity may cause spatial hole burning near the output facet as illustrated in Fig. 14.11. The reduction of the quantum well carrier density 


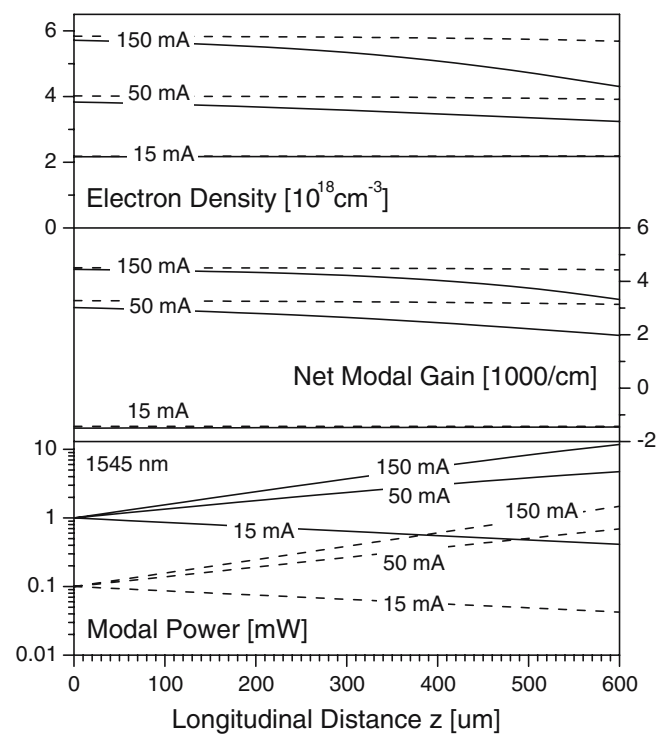

Fig. 14.10. Internal SOA parameters vs. longitudinal distance $(\mu \mathrm{m})$ at $1545 \mathrm{~nm}$ wavelength. Top: quantum well electron density; middle: net modal gain; bottom: light power. The SOA current is given as parameter and the fiber input power is $0.4 \mathrm{~mW}$ (dashed) and $4 \mathrm{~mW}$ (solid), respectively (at 15-mA injection current, the modal gain is negative and the SOA partially absorbs the input signal).

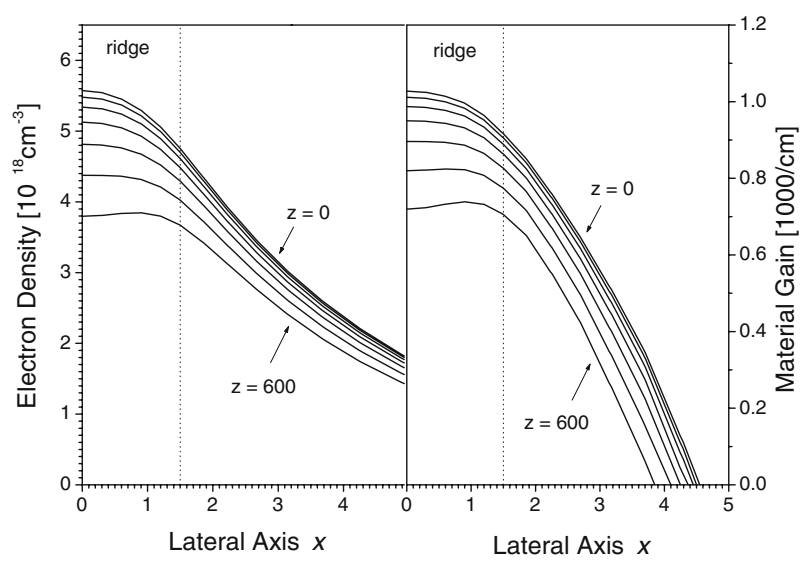

Fig. 14.11. Internal SOA parameters vs. lateral distance $(\mu \mathrm{m})$ with the longitudinal position $z / \mu \mathrm{m}$ in steps of $100 \mu \mathrm{m}$ as parameter (current $=150 \mathrm{~mA}$, fiber power $=4 \mathrm{~mW}$, wavelength $=1545 \mathrm{~nm})$. Left: quantum well electron density; right: material gain. 


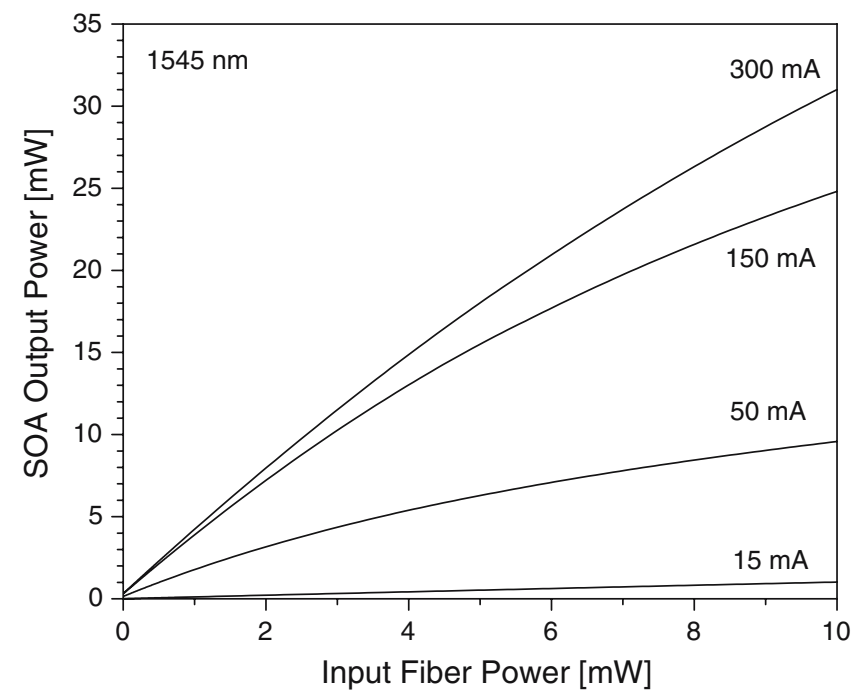

Fig. 14.12. Output power vs. input power for different SOA currents.

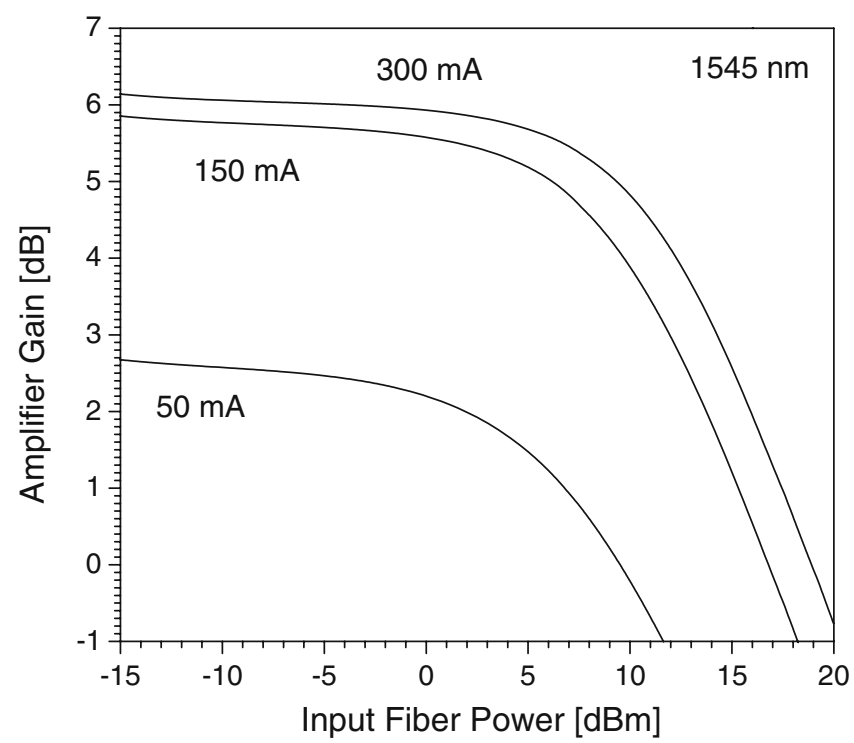

Fig. 14.13. Amplifier gain vs. input power for different SOA currents. 
with higher power is the main reason for the nonlinear amplifier response shown in Fig. 14.12. The ratio of output to input power gives the amplifier gain, which is plotted in Fig. 14.13, including fiber coupling losses. At maximum material gain $(300 \mathrm{~mA})$, the maximum amplifier gain is about $4(6 \mathrm{~dB})$ and the $-3 \mathrm{~dB}$ saturation power is about $25 \mathrm{~mW}(14 \mathrm{dBm})$.

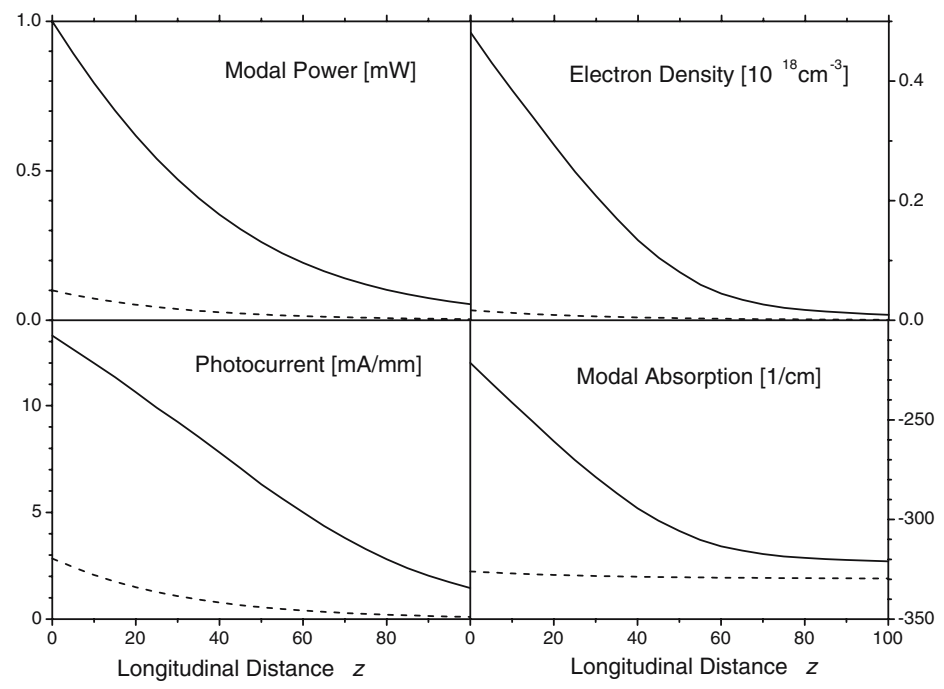

Fig. 14.14. Longitudinal variations $(z / \mu \mathrm{m})$ within the waveguide photodetector as calculated at $1545-\mathrm{nm}$ wavelength and $1-\mathrm{V}$ reverse bias for $1-\mathrm{mW}$ (solid) and 0.1-mW (dashed) input power.

\subsubsection{Photodetector}

The waveguide photodetector (WPD) is coupled monolithically to the preamplifier, and we neglect any optical coupling loss here. The WPD length is $100 \mu \mathrm{m}$ in our simulation. Detector saturation effects are mainly related to the accumulation of photogenerated carriers within the quantum wells. Such accumulation depends on the local light power and therefore on the longitudinal position $z$ within the detector. Figures 14.14 and 14.15 , respectively, show longitudinal and vertical variations within the detector at two different input power levels. At low input power $(0.1 \mathrm{~mW}$, dashed lines), the quantum well carrier density remains low and the band-to-band absorption is 
almost constant in longitudinal direction. The photocurrent $I_{\mathrm{ph}}(z)$ decays with light penetration depth due to the reduced modal power. At higher input power ( $1 \mathrm{~mW}$, solid lines), the photogenerated carriers pile up near the front and lead to a reduction of the band-to-band absorption. Therefore, the light penetrates deeper into the WPD and about $5 \%$ of the light power remains undetected (light reflection at the rear facet is neglected). The amount of undetected light increases with higher input light, leading to a nonlinear detector response.

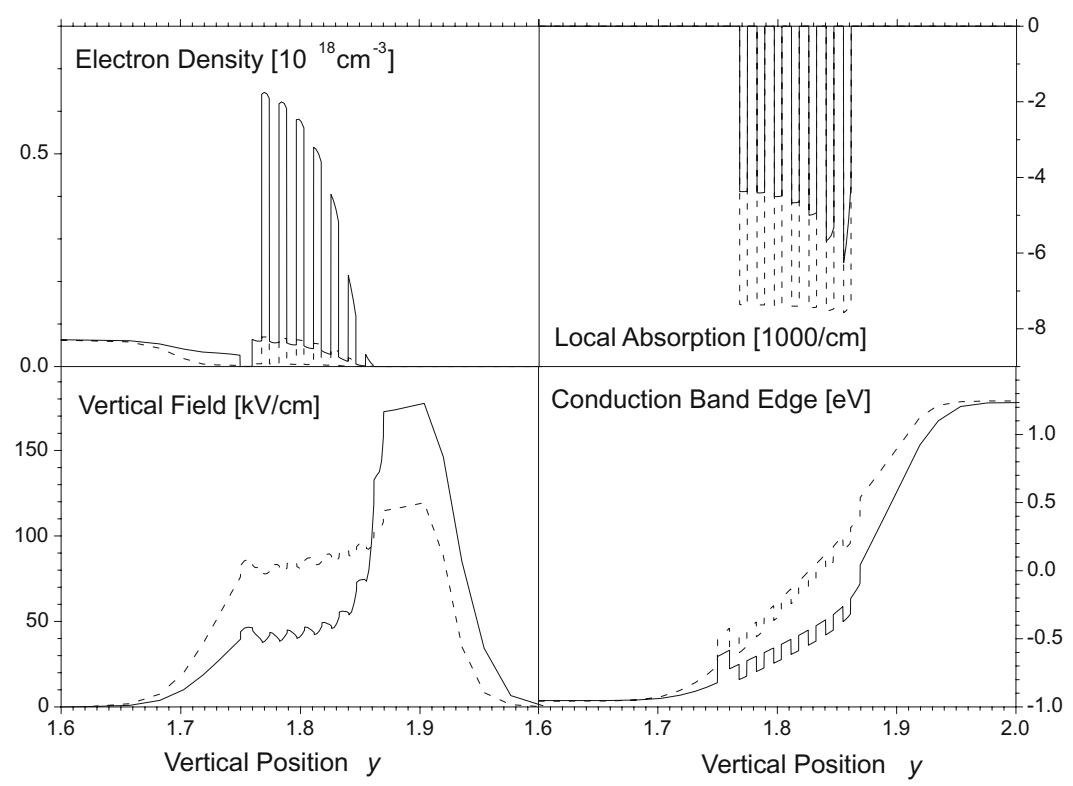

Fig. 14.15. Vertical variations in the center of the detector $(y / \mu \mathrm{m}, x=0, z=0)$ for $1-\mathrm{mW}$ (solid) and 0.1-mW (dashed) input power $(1545 \mathrm{~nm}$, reverse bias $=1 \mathrm{~V})$.

Figure 14.16 plots calculated detector response characteristics. There is a significant dependence on the input wavelength, which corresponds to the strong wavelength dependence of the absorption spectra shown in Fig. 14.6. At $1545 \mathrm{~nm}, 1-\mathrm{V}$ reverse bias, and 1-mW input power, the total quantum efficiency is $75 \%$, which indicates that about $20 \%$ of the photogenerated carriers are lost in recombination mechanisms without contributing to the photocurrent. The differential quantum efficiency is $60 \%$, and it further decreases with increasing input power.

The accumulation of quantum well carriers leads to a partial screening of the electrostatic field within the active region (Fig. 14.15), hindering the carrier transport to the contacts. Such screening can be reduced by applying a larger reverse electric field. The dashed line in Fig. 14.16 is calculated for 3$\mathrm{V}$ reverse bias, and it shows less nonlinearity than the low-bias characteristic. 


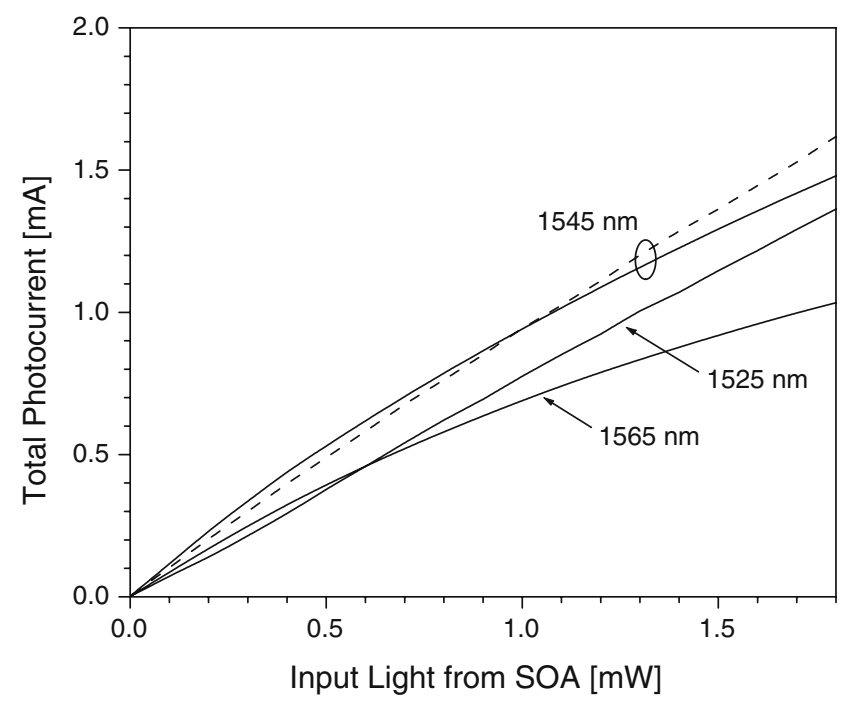

Fig. 14.16. Photodetector response for different wavelengths and for a reverse bias of $1 \mathrm{~V}$ (solid) and $3 \mathrm{~V}$ (dashed).

This leads to an improved differential quantum efficiency of $70 \%$ at 1-mW input power. However, the significant saturation calculated for our quantum well detector at relatively low input power may require the use of FranzKeldysh-type detectors in the wavelength converter. Such photon absorption by the reverse-biased waveguide layer shows less saturation effects due to the missing carrier confinement [2].

\subsubsection{Sampled-Grating DBR Laser}

A sampled grating is a conventional DBR grating with grating elements removed in a periodic fashion [8]. Such sampling leads to reflection spectra with periodic peaks (Fig. 14.17). As the figure shows, the periods of the two reflectivity spectra are slightly mismatched. Lasing occurs at that pair of maxima that is aligned. Each spectrum can be shifted by inducing small refractive index changes in the mirror, thereby changing the emission wavelength by aligning a different pair of reflection peaks. This Vernier tuning mechanism allows for six to eight times the tuning range that is achieved from the index change alone [20]. An overview of the different wavelength tuning mechanisms employed in semiconductor lasers can be found in [21].

Our example device contains five sections (Table 14.3) [9]. The rear mirror employs 12 periods of 6 - $\mu \mathrm{m}$-long grating bursts with $46-\mu \mathrm{m}$ period length. 


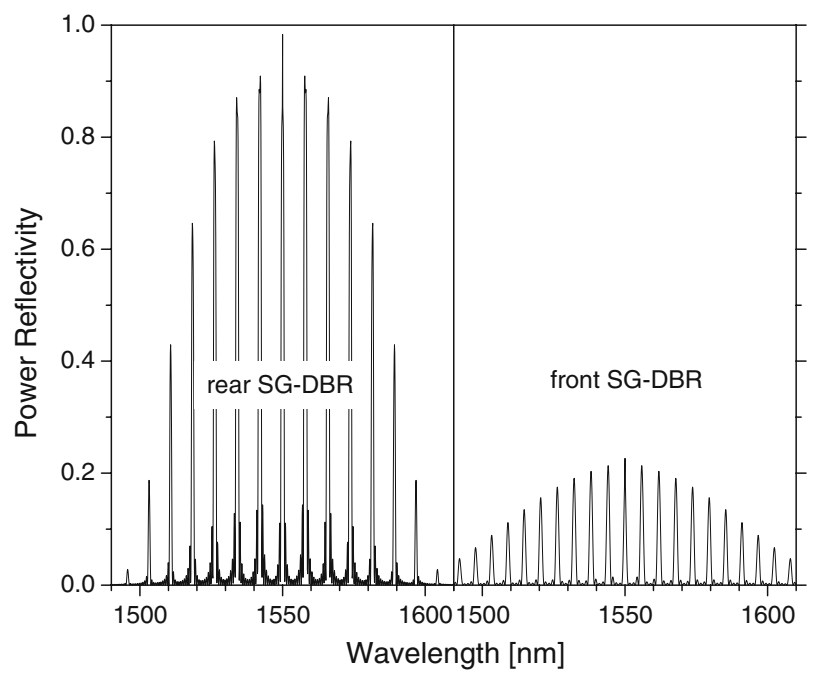

Fig. 14.17. Reflectivity spectra of the two SG-DBR mirrors.

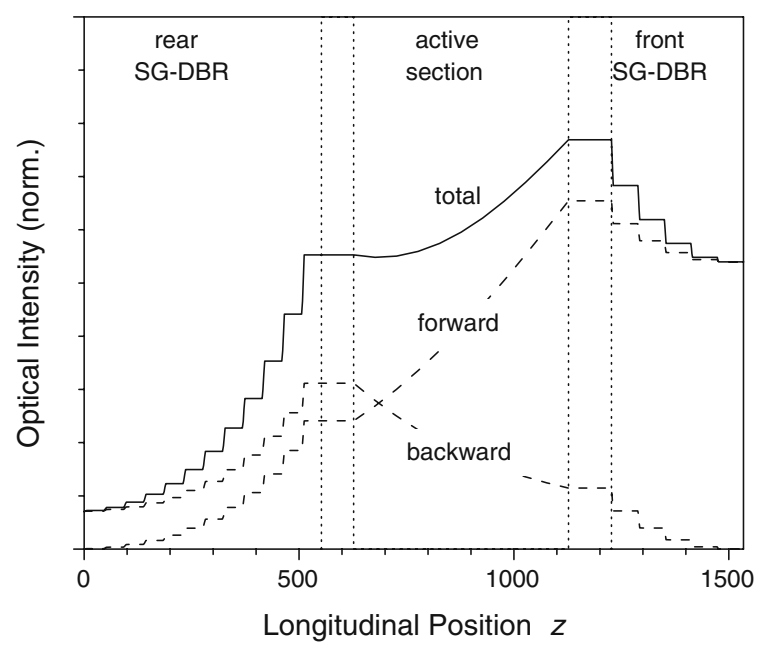

Fig. 14.18. Longitudinal intensity profile $(z / \mu \mathrm{m})$ within the SG-DBR laser for forward and backward traveling wave (dashed) and total intensity (solid). 
Table 14.3. Longitudinal Sections of the SG-DBR Laser $(L-$ section length, $\kappa$ - optical coupling coefficient of each grating burst).

\begin{tabular}{lll}
\hline & $\begin{array}{l}L \\
(\mu \mathrm{m})\end{array}$ & $\begin{array}{l}\kappa \\
(1 / \mathrm{cm})\end{array}$ \\
\hline Rear SG-DBR & 552 & 250 \\
Phase tuning & 75 & 0 \\
Active & 500 & 0 \\
Gain lever & 100 & 0 \\
Front SG-DBR 307.5 & 250
\end{tabular}

The front mirror has 5 periods of 4 - $\mu$ m-long grating bursts with $61.5-\mu \mathrm{m}$ period length. Both the SG-DBR reflectivity spectra are plotted in Fig. 14.17. The phase section is used to fine-tune the wavelength. The active section is used for DC pumping of the laser. The gain lever section is connected to the photodiode for direct laser modulation. Figure 14.18 shows the optical intensity along the longitudinal axis without photocurrent. Both the forward and the backward traveling wave gain intensity in the active section according to (14.2). Within the SG-DBR sections, each grating burst reflects part of the optical wave and causes a stepwise change in intensity.

Figure 14.19 gives the simulated light-current (LI) characteristics of the SG-DBR laser for two different emission wavelengths. At $1550 \mathrm{~nm}$, the threshold current is $26 \mathrm{~mA}$ and the slope efficiency $39 \%$. Due to the lower reflectivity at $1526 \mathrm{~nm}$, threshold gain and quantum well carrier density rise, giving an increased threshold current of $42 \mathrm{~mA}$ for this wavelength. The slope efficiency drops to $33 \%$, which is partially caused by the stronger differential change of the Auger recombination. This phenomenon is related to the nonuniform carrier distribution among the quantum wells, which leads to increasing recombination with increasing current, even if the total quantum well carrier density is constant [22]. Current adjustment is required to maintain the same DC power at different wavelengths. Postamplification helps to enhance both the slope efficiency and the signal power. The rear emission is also shown for comparison (dashed lines), it can be used to monitor the output signal. The laser emission spectrum is plotted in the insets of Fig. 14.19 for 13-mW output power. The side-mode suppression ratio (SMSR) is almost $40 \mathrm{~dB}$ in both cases, showing that the large tuning range of SG-DBR lasers can be combined with high SMSR.

Here, we cover SG-DBR lasers only briefly because a more detailed discussion and simulation of SG-DBR lasers is given in Chapt. 6 . 


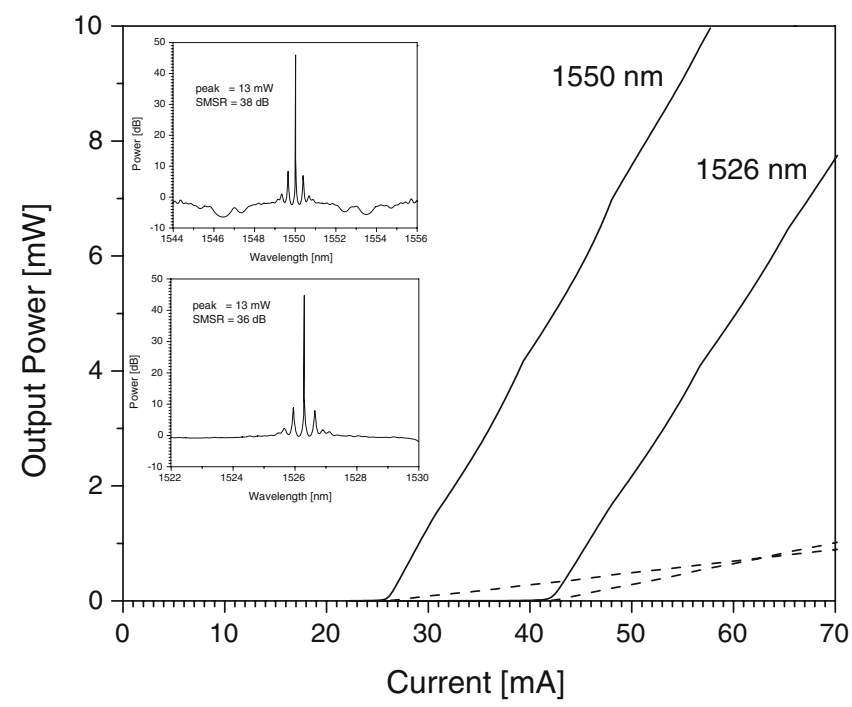

Fig. 14.19. Light vs. current characteristics for two laser wavelengths (solid front emission, dashed - rear emission); the insets give the emission spectrum for both cases, including the SMSR at 13-mW front output power.

\subsection{Summary}

We have demonstrated the inclusion of many-body theory into the full device simulation of all components comprising the monolithic wavelength converter. This allows for a more realistic prediction of the device performance at different wavelength. The detailed analysis of microscopic saturation mechanisms helps to improve the design of future converter generations.

\section{Acknowledgment}

This work was partially supported by the Semiconductor Research Corporation and by the Intel Corporation. The authors are grateful to Dr. John Hutchinson, Jeff Henness, Anna Tauke Pedretti, and Prof. Larry Coldren for valuable discussions and measurements. We also acknowledge fruitful discussions with Prof. Stephan W. Koch.

\section{References}

1. J. S. B. Yoo: IEEE J. Lightwave Technol. 14, 955 (1996) 
2. J. Hutchinson, J. Barton, M. Masanovic, M. Sysak, J. Henness, L. Johansson, D. Blumenthal, and L. A. Coldren: Monolithically integrated InP-based tunable wavelength conversion. In: Physics and Simulation of Optoelectronic Devices XII SPIE Proceedings 5349 (The International Society for Optical Engineering, Bellingham 2004)

3. PICS3D by Crosslight Software Inc., Burnaby, Canada (http://www.crosslight.com)

4. Nonlinear Control Strategies Inc., Tucson, AZ (http://www.nlcstr.com)

5. J. Hader, J. V. Moloney, S. W. Koch, and W. W. Chow: IEEE J. Select. Topics Quantum Electron. 9, 688 (2003)

6. J. Hutchinson, J. Henness, L. Johansson, J. Barton, M. Masanovic, and L. A. Coldren: $2.5 \mathrm{~Gb} / \mathrm{sec}$ wavelength conversion using monolithically-integrated photodetector and directly modulated widely-tunable SGDBR laser. In: Proc. 16th LEOS Annual Meeting Lasers and Electro-Optics Society, Piscataway, NJ (2003)

7. B. Mason, J. Barton, G. A. Fish, L. A. Coldren, and S. P. DenBaars: IEEE Photon. Technol. Lett. 12, 762 (2000)

8. V. Jayaraman, Z. M. Chuang, and L. A. Coldren: IEEE J. Quantum Electron. 29, 1824 (1993)

9. J. Piprek, N. Trenado, J. Hutchinson, J. Henness, and L. A. Coldren: 3D Simulation of an Integrated Wavelength Converter. In: Physics and Simulation of Optoelectronic Devices XII SPIE Proceedings 5349 (The International Society for Optical Engineering, Bellingham 2004)

10. C. Henry, R. Logan, F. R. Merritt, and J. P. Luongo: IEEE J. Quantum Electron. 19, 947 (1983)

11. S. Adachi: Physical Properties of III-V Semiconductor Compounds (Wiley, New York 1992)

12. S. L. Chuang: Physics of Optoelectronic Devices (Wiley, New York 1995)

13. J. Piprek, P. Abraham, and J. E. Bowers: IEEE J. Quantum Electron. 36, 366 (2000)

14. J. Henness, unpublished

15. W. W. Chow and S. W. Koch: Semiconductor-Laser Fundamentals (Springer, Berlin 1999)

16. I. Vurgaftman, J. R. Meyer, and L. R. Ram-Mohan: J. Appl. Phys. 89, 5815 (2001)

17. M. Sotoodeh, A. H. Khalid, and A. A. Rezazadeh: J. Appl. Phys. 87, 2890 (2000)

18. S. Seki, W. W. Lui, and K. Yokoyama: Appl. Phys. Lett. 66, 3093 (1995)

19. J. Piprek: Semiconductor Optoelectronic Devices: Introduction to Physics and Simulation (Academic Press, San Diego, 2003)

20. L. A. Coldren: IEEE J. Select. Topics Quantum Electron. 6, 988 (2000)

21. L. A. Coldren, G. A. Fish, Y. Akulova, J. S. Barton, L. Johansson, and C.W. Coldren: IEEE J. Lightwave Technol. 22, 193 (2004)

22. J.Piprek, P. Abraham, and J. E. Bowers: Appl. Phys. Lett. 74, 489 (1999) 